\title{
Painful pretibial pseudocyst at bioabsorbable interference screw aperture two years after anterior cruciate ligament reconstruction
}

Michael Xuanrong $\underline{\text { Shen }}^{1}$, MBBS, SS $\underline{\text { Sathappan }}{ }^{1}$, MBChB, FRCSE

\begin{abstract}
We report the case of a patient with a painful subcutaneous nodule, measuring $13 \mathrm{~mm} \times 17 \mathrm{~mm}$, at the pretibial graft aperture site, which presented two years after a successful anterior cruciate ligament reconstruction with an autologous hamstring graft. A bioabsorbable poly-L-lactide interference screw was used for graft fixation at the tibial aperture. The patient underwent surgical excision of the lesion and curettage at the tunnel aperture. Grossly, extruded fragments of the screw and a thick pseudocapsule of surrounding tissue were excised. There was no communication between the tunnel aperture and the knee joint. The graft was also intact. Histological analysis revealed fragments of the bioabsorbable material in association with fibrous and granulomatous chronic inflammatory cells. This was consistent with a foreign body reaction. The patient subsequently recovered and resumed preinjury level of activity. To the best of our knowledge, this is the first report describing a nodular granulomatous type reaction to foreign bioabsorbable poly-L-lactide screw material subsequent to an anterior cruciate reconstruction surgery.
\end{abstract}

Keywords: bioabsorbable interference screw, granuloma, pseudocyst, polylactic acid

\section{INTRODUCTION}

Bioabsorbable interference screws have increasingly replaced metallic screws as the more advantageous option for tibial graft fixation in anterior cruciate ligament $(\mathrm{ACL})$ reconstructions. Better postoperative imaging, (1) obviated need for screw removal ${ }^{(2)}$ and decreased risk of graft laceration ${ }^{(3)}$ have made bioabsorbable interference screws a common preference. Even so, a meta-analysis of eight studies by Emond et al comparing the results of $\mathrm{ACL}$ reconstruction using metallic screws versus $\mathrm{ACL}$ reconstruction using bioabsorbable screws showed no statistically significant differences in the mechanical and clinical outcomes of the two groups. ${ }^{(4)}$ More importantly, comparative data on the complications seen in the groups of patients who underwent $\mathrm{ACL}$ using either type of screws is lacking.

There have been emerging reports to suggest that the use of bioabsorbable screws are not free from a unique set of complications. For instance, Konan and Haddad, (2) who comprehensively reviewed 28 sporadic cases related to polyL-lactide screws, found that implant breakage during surgery, postsurgical infection and implant migration into the knee joint were cogent, worrisome complications associated with its use. The formation of acutely painful solitary pretibial cysts at the external tibial graft aperture has been reported in three other patients in the literature. ${ }^{(5,6)}$ Busfield and Anderson, ${ }^{(5)}$ in their report on pretibial abscess after $A C L$ reconstruction using poly-L-lactide bioabsorbable interference screws in two patients 3 years and 18 months after surgery, noted that the cysts contained $8 \mathrm{~mL}$ and $20 \mathrm{~mL}$ of chalky sterile fluid, respectively. Martinek and Friederich ${ }^{(6)}$ also described a similar occurrence in a patient eight months after surgery using a poly-D,L-lactide interference screw. In the aforementioned cases, fragments of screw material were visible on histology, but inflammatory or granulomatous tissue reactions were not present. ${ }^{(5,6)}$

Herein, we report the case of a patient who presented with a firm and painful subcutaneous nodule, measuring $13 \mathrm{~mm} \times$ $17 \mathrm{~mm}$, over the external tibial graft aperture approximately two years after $\mathrm{ACL}$ reconstruction. To the best of our knowledge, this is the first report of a firm nodular tissue reaction over the tibial screw fixation site.

\section{CASE REPORT}

A 21-year-old man initially presented with multiple ligamentous injuries to his left knee, resulting from an injury in the valgus flexion during judo practice. Magnetic resonance (MR) imaging demonstrated a complete tear of the $\mathrm{ACL}$, high-grade partial tear of the medial collateral ligament and peripheral tear of the lateral meniscus. Surgery was advised due to knee instability, locking and the patient's significantly limited function. Meniscal debridement was performed, as well as ACL reconstruction using semitendinosus and gracilis tendon grafts, with tibial fixation achieved using a $10 \mathrm{~mm} \times 28 \mathrm{~mm}$ poly-L-lactide Bio-Interference Screws (AR-1400TB; Arthrex Inc, Naples, FL, USA).

The patient was ambulated on crutches, with weight bearing permitted as tolerated. On postoperative Day 14, his

${ }^{1}$ Sports and Adult Reconstruction, Department of Orthopaedic Surgery, Tan Tock Seng Hospital, Singapore

Correspondence: Dr Michael Shen Xuanrong, Resident, Department of Orthopaedic Surgery, Tan Tock Seng Hospital, 11 Jalan Tan Tock Seng, Singapore 308433. sheny_mike@hotmail.com 

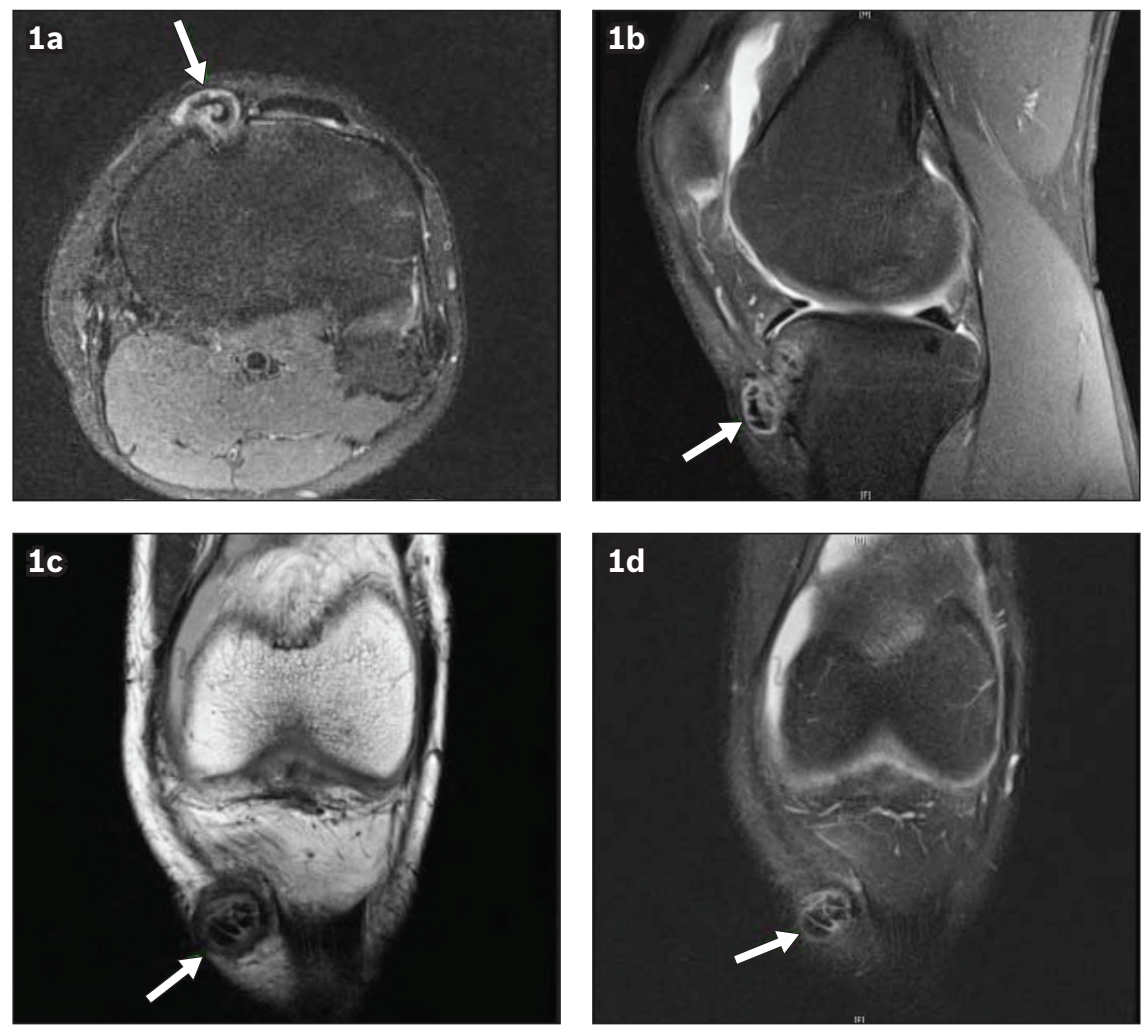

Fig. 1 Standard non-contrast MR imaging sequences show a $13 \mathrm{~mm} \times 17 \mathrm{~mm}$ nodular lesion (arrows) at the anteromedial aspect of the proximal tibia overlying the tibial tunnel aperture. (a) Axial proton density fat-saturation image; (b) sagittal proton density fat-saturation image; (c) coronal proton density image; and (d) coronal proton density fat-saturation image.

incision was inspected and found to be clean, dry and intact. Subsequently, the patient made a full recovery and successfully returned to pre-injury level of activity about five months after surgery. On examination of the knee one year post surgery, the patient showed full range of motion $\left(0^{\circ}\right.$ to $\left.125^{\circ}\right)$, no effusion and a negative Lachman's test. No signs or symptoms of infection, dehiscence or tissue reaction were noted.

Two years after surgery, however, the patient returned complaining of localised pain and tenderness, with the development of a firm nodule over the previous tibial incision site. The lesion was noted to have progressively enlarged since its onset two weeks ago, and was associated with pain that was of sufficient severity to impair daily activities. There were no other knee symptoms. The patient also did not notice any association with trauma, fever, chills/rigors or any similar lesions elsewhere.

On examination, a firm $13 \mathrm{~mm} \times 17 \mathrm{~mm}$ subcutaneous nodule was observed at the anteromedial aspect of the proximal tibia overlying the site of tibial graft fixation. The overlying skin was erythematous, but grossly intact. On proton density MR imaging, a lesion was demonstrated at the specified site, with features highly suggestive of a granuloma (Fig. 1). An intact screw could not be visualised on imaging, and therefore, its protrusion out of the tunnel was uncertain. Inflammatory markers at the time of presentation were not elevated.

The tender harvest site was reincised and explored, allowing visualisation of the subcutaneous nodular pseudocyst.
Following successful isolation, a majority of the lesional mass was found to be extraosseous, although a small proportion was also intraosseous, extending into the tibial tunnel. Following excision, dissection of the mass yielded minimal serous fluid centrally $(<1 \mathrm{~mL})$. Fragments of solid white crystalline particles $(20 \mathrm{~mm} \times 10 \mathrm{~mm} \times 10 \mathrm{~mm}$ in aggregate) lay enveloped within dense surrounding capsular tissue $(8 \mathrm{~mm} \times 15 \mathrm{~mm})$. The term 'pseudocyst' aptly described the tissue architecture of the lesion, as unlike a true cyst, the lesion had considerably more solid tissue component than cystic component. The remaining cavity leading into the tunnel was curetted and irrigated. Deeper osseous reconstitution was both visualised and confirmed on tactile probing. No residual screw material remained in the tunnel. There was also no evidence to suggest communication between the external aperture and knee joint.

Tissue samples sent for Gram staining and bacterial cultures were negative. Histopathological analysis revealed dense fibrous tissue in association with multiple, variably sized, white refractile particles. The foreign screw material was surrounded by multinucleated giant cells, exhibiting a histiocytic reaction with accompanying mild chronic inflammatory infiltrate. Overall findings were suggestive of a foreign body granulomatous type reaction. Following surgery, the patient's symptoms resolved and he rehabilitated well. Full premorbid level of activity was successfully resumed one month into recovery, and the patient was well at his most recent follow-up. 


\section{DISCUSSION}

Studies conducted on bioabsorbable polylactide for surgical implants examined the histological tissue response and rate of degradation of the polymer. The first published study was by Kulkarni et al in the 1960s..$^{(7)}$ These studies, conducted on poly-L-lactide powder implanted within animal tissue, did not suggest any toxic or tissue reactions relating to the polymer. Corroborating data clearly demonstrating the absence of inflammatory or foreign body reactions was again published in 1971 by Kulkarni et al. ${ }^{(8)}$

Structurally, polylactide is composed of lactic acid subunits strung and unified through ester bonds to form a polymer chain. Natural biodegradation reverses this process, yielding monomeric subunits that can then be oxidised to pyruvic acid and eliminated through the tricarboxylic acid cycle. ${ }^{(9)}$ The biologically inert end products of carbon dioxide and water seamlessly integrate into the cellular milieu. However, the rate of biodegradation and debris formation may occasionally exceed the metabolic limits of phagocytic tissue. This localised overload of debris was observed in the mid-1980s during the advent of bioabsorbable fracture fixation devices involving polylactide. A small series of case reports described intraosseous collections of sterile effusions with partially degraded screw material suspended in them. ${ }^{(10}$

The use of polylactide screws in $\mathrm{ACL}$ reconstruction has also yielded adverse reactions in a similar way. Busfield and Anderson, ${ }^{(5)}$ as well as Martinek and Friederich, ${ }^{(6)}$ have described the formation of sizeable cystic lesions at the tibial aperture, the contents of which included degraded chalky screw material suspended in sterile joint fluid, but no accompanying inflammatory tissue. Many other reports have also described deeper cystic collections within the tibial tunnel, even those communicating with the knee joint space. ${ }^{(11)}$ Tsuda et al, ${ }^{(11)}$ who described such a phenomenon as water channels, postulated very reasonable aetiological mechanisms such as noncongruent bone tendon interface (mismatched graft/tunnel diameters), excessive graft motion, early graft necrosis and tunnel expansion.

Mechanisms for biodegradation, debris phagocytosis and osseous reconstitution differ depending on the surrounding cellular milieu. Intraosseous reactions have been generally reported as cystic effusions, while extraosseous reactions are comparatively sporadic with heterogeneous presentations. More recent studies of polylactide describe its potential ability to activate the complement system and trigger adverse immune reactions. ${ }^{(12)}$ However, such evidence is still controversial. The biggest case series of pretibial reactions to date is by Gonzalez-Lomas et $\mathrm{al}^{\left({ }^{(12)}\right.}$ who documented seven patients with cystic reactions at the tibial aperture in a cohort of 140 patients who underwent primary $\mathrm{ACL}$ reconstructions. Among these seven patients, four had some form of cystic collection in communication with the joint space, while the remaining three patients had isolated cystic collections with some evidence of inflammatory reactions. A foreign body reaction was postulated as the potential aetiological mechanism for the development of these cysts. In our patient, however, an isolated granulomatous tissue response was seen to the polylactide screw material following ACL surgery. Our findings are therefore relatively less common among the various adverse tissue reactions usually seen in such patients.

Recent literature chronicling the in situ degradation profile of polylactide has also shed some light on its true properties. In 2012, Sprowson et al ${ }^{(13)}$ completed a ten-year MR imaging observational study of patients with bioabsorbable polylactide interference screws used for tibial fixation and found that no resorption had occurred after four years in any of the patients. During the ten-year follow-up period, however, half of their patients displayed small cystic collections within the tibial tunnel. The study demonstrated that polylactide screws in situ took approximately twice as long to resorb compared to historical in vitro data. ${ }^{(14)}$ In other words, the biodegradation profile of polylactide may be more ambiguous than popularly believed. Cyst formation within the tibial tunnel, cyst formation at the aperture and granulomatous tissue reaction at the screw head-subcutaneous tissue interface are specific presentations related to abnormal screw degradation and resorption. ${ }^{(15)}$

We postulate that a combination of factors may contribute to such adverse reactions. A localised overload of debris formation from uncoordinated biodegradation, articular communication and some element of abnormal graft motion may explain cystic complications following the use of polylactide screws. Immunological reaction and biological incompatibility (e.g. in patients with lactate dehydrogenase insufficiency) are also contributory and may explain the inflammatory component of these reactions.

Although these complications are rare, it is important for surgeons to be cognisant of the various adverse tissue reactions. The mechanisms and risk factors for these complications are not well studied and deserve more attention. Individual-toindividual variation in biodegradation and immunological mechanisms are important areas for future research. Despite its perceived advantages, bioabsorbable screws as an alternative to metal screws for $\mathrm{ACL}$ reconstruction presents a unique set of complications and can severely impact outcomes for selected patients who have bioabsorbable screws as surgical implants. Based on our experience and the literature analysis, we recommend that all symptomatic, pretibial lesions presenting within four years of $\mathrm{ACL}$ reconstruction undergo operative exploration and excision, as outcomes are excellent with expedient recovery to function once these inflammatory lesions are excised.

To summarise, the search for the ideal material for surgical implants continues, and future development should be aimed at engineering the best material properties and degradation characteristics to improve fixation and osseous reconstitution, while averting foreign body response in all individuals. ${ }^{(16)}$ 


\section{REFERENCES}

1. Shellock FG, Mink JH, Curtin S, Friedman MJ. MR imaging and metallic implants for anterior cruciate ligament reconstruction: assessment of ferromagnetism and artifact. J Magn Reson Imaging 1992; 2:225-8.

2. Konan S, Haddad FS. A clinical review of bioabsorbable interference screws and their adverse effects in anterior cruciate ligament reconstruction surgery. Knee 2009; 16:6-13.

3. Shen C, Jiang SD, Jiang LS, Dai LY. Bioabsorbable versus metallic interference screw fixation in anterior cruciate ligament reconstruction: a meta-analysis of randomized controlled trials. Arthroscopy 2010 26:705-13.

4. Emond CE, Woelber EB, Kurd SK, Ciccotti MG, Cohen SB. A comparison of the results of anterior cruciate ligament reconstruction using bioabsorbable versus metal interference screws: a meta-analysis. J Bone Joint Surg Am 2011; 93:572-80.

5. Busfield BT, Anderson LJ. Sterile pretibial abscess after anterior cruciate reconstruction from bioabsorbable interference screws: a report of 2 cases. Arthroscopy 2007; 23:911.e1-4.

6. Martinek V, Friederich NF. Tibial and pretibial cyst formation after anterior cruciate ligament reconstruction with bioabsorbable interference screw fixation. Arthroscopy 1999; 15:317-20.

7. Kulkarni RK, Pani KC, Neuman C, Leonard F. Polylactic acid for surgical implants. Arch Surg 1966; 93:839-43.
8. Kulkarni RK, Moore EG, Hegyeli AF, Leonard F. Biodegradable poly(lactic acid) polymers. J Biomed Mater Res 1971; 5:169-81.

9. Böstman OM, Pihlajamäki HK. Late foreign-body reaction to an intraosseous bioabsorbable polylactic acid screw. A case report. J Bone Joint Surg Am 1998; 80:1791-4.

10. Böstman OM, Pihlajamäki HK. Adverse tissue reactions to bioabsorbable fixation devices. Clin Orthop Relat Res 2000; 216-27.

11. Tsuda E, Ishibashi $Y$, Tazawa $K$, et al. Pretibial cyst formation after anterior cruciate ligament reconstruction with a hamstring tendon autograft. Arthroscopy 2006; 22:691.e1-6.

12. Gonzalez-Lomas G, Cassilly RT, Remotti F, Levine WN. Is the etiology of pretibial cyst formation after absorbable interference screw use related to a foreign body reaction? Clin Orthop Relat Res 2011; 469:1082-8.

13. Sprowson AP, Aldridge SE, Noakes J, Read JW, Wood DG. Bio-interference screw cyst formation in anterior cruciate ligament reconstruction -- 10-year follow up. Knee 2012; 19:644-7.

14. Migliaresi C, Fambri L, Cohn D. A study on the in vitro degradation of poly(lactic acid). J Biomater Sci Polym Ed 1994; 5:591-606.

15. Brager MA, Traina SM, Parker AW. Pretibial cyst following anterior cruciate ligament reconstruction using hamstring autografts. Orthopedics 2002; 25:79-82.

16. Ambrose CG, Clanton TO. Bioabsorbable implants: review of clinical experience in orthopedic surgery. Ann Biomed Eng 2004; 32:171-7. 